\section{COVID-19 collateral damage: Alcohol rub dermatitis as an emerging problem}

To the Editor: COVID-19 has spread across the globe and was declared a global pandemic by the World Health Organization (WHO) in March 2020. ${ }^{[1]}$ The WHO and the Centers for Disease Control and Prevention (CDC) recommended that the best way to prevent infection is via social distancing, utilising cloth masks, and frequent and proper handwashing and surface decontamination. ${ }^{[1]}$ These recommendations have resulted in an exponential rise in the use of alcohol-based hand sanitisers, which has led to a rise in the incidence of hand eczema in healthcare workers as well as the general population. ${ }^{[2]}$ Certain individuals are at increased risk, for example those with atopic eczema with their inherent barrier dysfunction (Fig. 1). ${ }^{[3]}$

In a study from Hubei Province in China, 434 healthcare workers were interviewed, with 321 (74\%) reporting that they sanitised their hands more than 10 times per day. Of those healthcare workers, 246 (76.6\%) reported symptoms of dermatitis, with the most common being irritant contact dermatitis and less commonly allergic contact dermatitis. ${ }^{[1]}$ A study from Milan reported that during the period 9 March - 4 May 2020, there were 24 new cases of hand eczema in the general population related to the use of alcohol-based sanitisers. ${ }^{[4]}$ Issues with alcohol-based hand sanitisers include the use of non-standard formulas, meaning that methanol is used instead of ethanol, types of alcohol used, such as isopropyl alcohol v. ethanol, and varying amounts of alcohol being used $(60-80 \%)$. $^{[5]}$

Apart from the discomfort of dry, itchy skin, the breaks in the skin can serve as a point of entry for COVID-19 because of the rich expression of viral receptors in the blood vessels of the epidermal and dermal structures. Additionally, alcohol rub dermatitis can become a debilitating and painful disease resulting in work absenteeism, which can severely impair the delivery of essential services. $^{[6]}$

Painful, dry skin could also inadvertently decrease hand hygiene compliance, which further increases the risk of disease transmission in these individuals. ${ }^{[1]}$ Prevention is therefore essential.

A lesser-known complication associated with a disrupted skin barrier is the increased potential to develop allergic contact dermatitis owing to the ease of access of allergens to the immune system. Sensitisation follows, and then an increased chance of a type IV hypersensitivity reaction in the form of an eczematous reaction ${ }^{[7]}$ that can affect quality of life, as well as causing occupational difficulties. ${ }^{[8]}$

Hand sanitiser containing $60 \%$ alcohol, with $1.45 \%$ glycerol as a humectant, has been recommended by the WHO to protect healthcare workers, and now the frequently sanitising public, from the complications of excessive alcohol hand rub use. The minimum amount of glycerol required to decrease the risk of dermatitis is not known, although studies have shown that even lower levels than the recommended $1.45 \%$ were effective to protect the skin from excessive drying. ${ }^{[9]}$

Further recommendations include the use of an emollientcontaining hand cream immediately after sanitising. ${ }^{[6,7]}$ Although this is a useful measure to prevent alcohol rub dermatitis, a study showed that only $\sim 1$ in 5 healthcare workers applied the cream after using alcohol hand rub. ${ }^{[6]}$

Sanitising stations are located everywhere in hospitals and public areas and have been an important means of combating the spread of COVID-19. However, moisturisers are few and far between, hence the increased incidence of alcohol rub dermatitis during the pandemic.

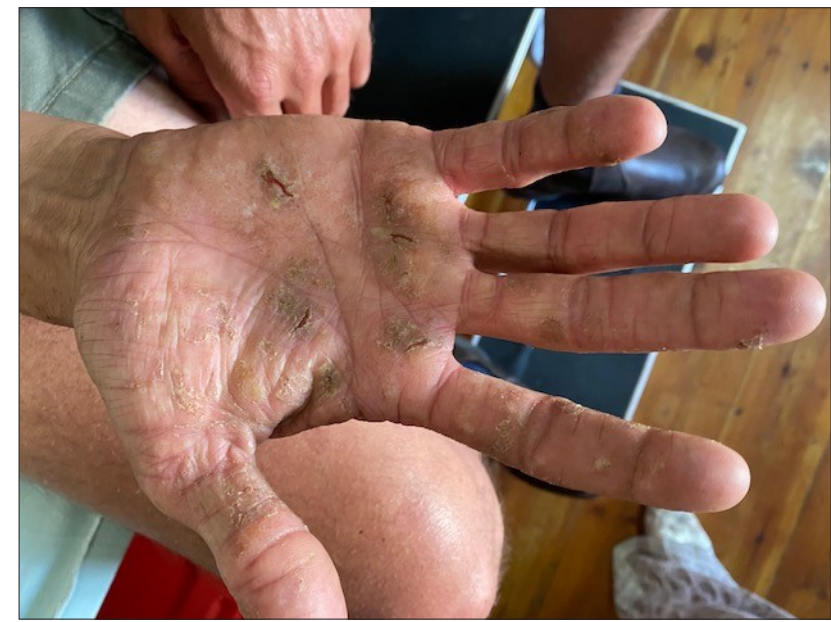

Fig. 1. Chronic hand dermatitis with hyperkeratotic plaques and fissures.

Adequate skin care could increase hand hygiene compliance ${ }^{[1]}$ and should therefore be prioritised, especially among healthcare workers. Furthermore, preventive measures are critical to allay the next, albeit completely different, wave: that of allergic contact dermatitis, another unintended health consequence ${ }^{[10]}$ of COVID-19.

\section{S M Alves, A J Arendse}

5th-year medical students, Division of Dermatology, Faculty of Medicine and Health Sciences, Stellenbosch University, Cape Town, South Africa

\section{S M H Kannenberg}

Consultant dermatologist, Division of Dermatology, Faculty of Medicine and Health Sciences, Stellenbosch University and Tygerberg Academic Hospital, Cape Town, South Africa

surethak@sun.ac.za

1. MacGibeny MA, Wassef C. Preventing adverse cutaneous reactions from amplified hygiene practices during the COVID-19 pandemic: How dermatologists can help through anticipatory guidance. Arch Dermatol Res 2020 (epub 9 May 2020). https://doi.org/10.1007/s00403-020-02086-x

2. Singh M, Pawar M, Bothra A, Choudhary N. Overzealous hand hygiene during the COVID 19 pandemic causing an increased incidence of hand eczema among general population. J Am Acad Dermatol 2020;83(1):e37-e41. https://doi.org/10.1016/j.jaad.2020.04.047

3. Ruff SMD, Engebretsen KA, Zachariae C, et al. The association between atopic dermatitis and hand eczema: A systematic review and meta-analysis. Br J Dermatol 2018;178(4):879-888. https://doi org/10.1111/bjd.16147

4. Giacalone S, Bortoluzzi P, Nazzaro G. The fear of COVID-19 infection is the main cause of the new diagnoses of hand eczema: Report from the frontline in Milan. Dermatol Ther 2020;33(4):el3630. https://doi.org/10.1111/dth.13630

5. Emami A, Javanmardi F, Keshavarzi A, Pirbonyeh N. Hidden threat lurking behind the alcohol sanitisers in COVID-19 outbreak. Dermatol Ther 2020;33(4):e13627. https://doi.org/10.1111/ dth. 13627

6. Patruno C, Fabbrocini G, Stingen L, Napolitano M. The role of occupational dermatology in the COVID-19 outbreak. Contact Dermatitis 2020;83(2):174-175. https://doi.org/10.1111/cod.13568 (accessed 30 August 2020)

(accessed 30 August 2020) can cause hand dermatitis: Management tips. Cureus 2020;12(4):e7506. https://doi.org/10.7759/ cureus. 7506

8. Kadyk DL, McCarter K, Achen F, Belsito DV. Quality of life in patients with allergic contact dermatitis. J Am Acad Dermatol 2003;49(6):1037-1048. https://doi.org/10.1016/S0190-9622(03)02112-1

9. Menegueti MG, Laus AM, Ciol MA, et al. Glycerol content within the WHO ethanol-based handrub formulation: Balancing tolerability with antimicrobial efficacy. Antimicrob Resist Infect Control 2019;8:109. https://doi.org/10.1186/s13756-019-0553-z

10. Burger R, Nkonki L, Rensburg R, Smith A, van Schalkwyk C. Examining the unintended health consequences of the COVID-19 pandemic in South Africa. Wave 1, National Income Dynamics Study (NIDS) - Coronavirus Rapid Mobile Survey (CRAM), 15 July 2020. https://cramsurveyorg wp-content/uploads/2020/07/Burger-examining-the-unintended-health-consequences pdf (accessed 4 September 2020). 